\title{
A 12-month prospective, observational study evaluating the impact of disease-modifying treatment on emotional burden in recently-diagnosed multiple sclerosis patients: The POSIDONIA study
}

\author{
Enrico Montanari ${ }^{\mathrm{a}, *}$, Mariarosa Rottoli ${ }^{\mathrm{b}}$, Davide Maimone ${ }^{\mathrm{c}}$, Paolo Confalonieri ${ }^{\mathrm{d}}$, Katrin Plewnia ${ }^{\mathrm{e}}$, \\ Maura Frigo ${ }^{\mathrm{f}}$, Ada Francia ${ }^{\mathrm{g}}$, Antonello Pala ${ }^{\mathrm{h}}$, Nunzia Alessandra Losignore ${ }^{\mathrm{i}}$, Paolo Ragonese ${ }^{\mathrm{j}}$, \\ Antonella Veneziano ${ }^{\mathrm{k}}$, on behalf of the POSIDONIA study group
}

a Azienda USL di Parma - Ospedale di Fidenza, Fidenza, Italy

b Centro Sclerosi Multipla, Ospedale Papa Giovanni XXIII, Bergamo, Italy

c Centro Sclerosi Multipla, U.O. Neurologia, Azienda Ospedaliera Garibaldi, Catania, Italy

d Centro Sclerosi Multipla, U.O Neurologia IV, Fondazione Istituto Neurologico Carlo Besta, Milano, Italy

e Ospedale Misericordia di Grosseto, Grosseto, Italy

${ }^{\mathrm{f}}$ Clinica Neurologica, Ospedale San Gerardo, Monza, Italy

g Centro Sclerosi Multipla Azienda Policlinico Umberto I, Roma, Italy

${ }^{\text {h }}$ U.O. di Neurologia Ospedaliera, Presidio Ospedaliero A. Segni, Ozieri, Italy

${ }^{\text {i } U . O ~ N e u r o l o g i a, ~ P r e s i d i o ~ O s p e d a l i e r o ~ D i m i c c o l i, ~ B a r l e t t a, ~ I t a l y ~}$

j Department of Experimental Biomedicine and Clinical Neuroscience, University of Palermo, Palermo, Italy

${ }^{\mathrm{k}}$ Medical Department, Teva Italia, Milano, Italy

\section{A R T I C L E I N F O}

\section{Article history:}

Received 26 October 2015

Received in revised form 25 January 2016

Accepted 18 February 2016

Available online 20 February 2016

\section{Keywords:}

Multiple sclerosis

Disease-modifying treatment

Emotional burden

Anxiety

Depression

\begin{abstract}
A B S T R A C T
Introduction: Depression and anxiety are common among patients with multiple sclerosis (MS) and are frequently present at the time of MS diagnosis.

Methods: POSIDONIA was a 12-month, observational, prospective study conducted in Italy to evaluate the impact of disease-modifying treatment (DMT) on emotional burden in patients with recently-diagnosed MS. The Hospital Anxiety and Depression Scale (HADS), specifically HADS anxiety (HADS-A) and depression (HADS-D) subscale scores, the Short-Form 36 Health Survey (SF-36) and the Impact of Event Scale - Revised (IES-R) were used to measure patient-reported outcomes. The Hamilton Depression Rating Scale (HDRS), HDRS-17, was used as a measure of healthcare provider-reported outcomes. The primary study outcome was change from baseline in feelings of anxiety and depression over 12 months (via HADS).

Results: Of 250 enrolled patients, 222 (88.8\%) completed the study. At baseline, mean HADS total, HADS-A and HADS-D subscale scores were within the normal range. There were no significant changes over time in mean HADS total and HADS-A and HADS-D subscale scores, although the subgroup of patients with baseline scores indicative of anxiety or depression tended to improve over time. Both the HDRS and IES-R total scores improved over time, but there were no statistically significant changes in SF-36.

Conclusion: In the patient population of the POSIDONIA study depression and anxiety were present in a minority of patients thus not allowing to detect the impact of starting DMT. However DMT appears to have a positive effect in patients with measurable anxiety or depression at baseline.
\end{abstract}

(c) 2016 Published by Elsevier B.V.

\section{Introduction}

Multiple sclerosis (MS) is a chronic and potentially highly disabling condition [1]. It may be responsible for considerable personal, social and economic consequences, and have a significant impact on healthrelated quality of life (HR-QOL) [1].

* Corresponding author at: Azienda USL di Parma - Ospedale di Fidenza, Via Don Enrico Tincati, 5, 43036 Fidenza, Italy.

E-mail address: emontanari@ausl.pr.it (E. Montanari).
Notably, depression and anxiety are common in patients with MS and occur substantially more often in patients with MS than in the general population [2,3]. The prevalence of depression among patients with MS has been estimated to be $11 \%$ to $50 \%$ [4-9], and anxiety to be $20 \%$ to $40 \%[4,5,8,9]$. Causes of depression and anxiety in this patient population include MS-related processes, genetic and environment-related predisposition, normal grieving and adjustment to loss [10,11]. Although depression and anxiety tend to worsen as physical disability increases [12], psychiatric comorbidities such as depression and anxiety are frequently present at the time of MS diagnosis $[2,3,8,13]$. 
Given that MS is characterized by unpredictable variations in symptoms, severity and progression [1], uncertainty about their illness is a common feeling in patients with MS, and may contribute to anxiety and depression. Recognizing and managing emotional needs is a key aspect of patient-centred care in MS [14]. Medication use may reduce uncertainty and distress, as patients may perceive that, through medication use, they are doing something to control the progression of their disease.

POSIDONIA (Prospective, Observational Study evaluating Impact of DMT treatment On the emotioNal burden in recently dIAgnosed multiple sclerosis patients) was a 12-month, prospective observational study that aimed to evaluate the impact of disease-modifying treatment (DMT) on emotional burden (changes in feelings of anxiety and depression) in patients with recently-diagnosed MS.

\section{Methods}

\subsection{Patients}

The POSIDONIA study was conducted in patients diagnosed with MS who were willing to be treated with DMT, following clinical practice. Eligible patients were required to have a confirmed and documented recent diagnosis of MS (as defined by revised McDonald criteria [15]), with a relapsing-remitting disease course, aged 18-65 years and had initiated DMT at the investigator's decision. In addition, they had to be able to sign and date a written informed consent form prior to entering the study and be willing and able to comply with the protocol requirements for the duration of the study. Patients who had received any previous treatment with any DMT were excluded, as were those who had used experimental treatment, investigational drugs or immunosuppressants (including mitoxantrone) prior to the baseline visit. Women who were pregnant or breastfeeding were also excluded, as were those with an insufficient ability to read, write, communicate and understand patient questionnaires.

\subsection{Study design}

This was an observational, prospective study conducted in 39 centres in Italy. The decision to utilise DMT was made by the treating physician following usual clinical practice. Patients were followed for 12 months, with data collected at baseline (week 0 , before treatment), and at two follow-up visits at 6 months ( \pm 2 weeks) [visit 2] and 12 months ( \pm 2 weeks) [visit 3]. A case report form designed specifically for the study was used by physicians to record sociodemographic and clinical variables (obtained by reviewing medical records or taking the patient's history). Sociodemographic variables included age, sex, and level of educational attainment. Clinical variables recorded were: date of first MS-related medical visit; duration of first MS symptoms; date of MS diagnosis confirmation; type of MS; currently prescribed DMT and dose of treatment; co-morbidities; co-medication; data on physical and neurological examination.

There were three patient-reported outcomes investigated in this study. The first utilized the Hospital Anxiety and Depression Scale (HADS), specifically looking at HADS anxiety (HADS-A) and depression (HADS-D) subscale scores, which range from 0 (no symptoms) to 21 (most severe symptoms). A HADS-A and HADS-D score of $\geq 8$ indicates a high risk of anxiety and depressive disorder in MS patients [16]. The Short-Form 36 Health Survey (SF-36) was also used. This comprises physical health scales (physical functioning, role-physical, bodily pain and general health) and mental health scales (vitality, social functioning, role-emotional and mental health); scores range from 0 (poor health) to 100 (optimal health). The third tool used was the Impact of Event Scale - Revised (IES-R), comprising 22 questions which examine the psychological stress of having MS by focusing on the intensity of thoughts and feelings relating to MS within the previous 7 days. Additionally, investigator-reported outcomes were assessed using the
Hamilton Depression Rating Scale (HDRS), HDRS-17, which assesses 17 items, with a maximum total score of 52; a total score of 0-7 is considered normal, whereas a total score of $\geq 18$ indicates substantial depression. Data on compliance, concomitant medication use, reported adverse events and reported serious adverse events were also collected.

The primary study objective was change from baseline in feelings of anxiety and depression over 12 months, assessed by HADS. HADS is a screening tool with robust psychometric properties, suitable for use in subjects with medical condition as it omits items assessing somatic symptoms, and is widely-used in MS patients. Secondary objectives were changes in feeling of depression, assessed by HDRS, healthrelated quality of life, assessed by SF-36, and disease-related psychological distress, assessed by IES-R.

\subsection{Study conduct}

All study participants provided written informed consent. The study adhered to the tenets of the Declaration of Helsinki and to International Guidelines for Ethical Review of Epidemiological Studies; it was conducted in accordance with national/local laws/regulations. The study protocol was approved by the Independent Ethics Committee at each participating site. The study was sponsored by Teva Italia.

\subsection{Statistical analysis}

The primary objective was to evaluate change in anxiety and depression in patients with recently-diagnosed MS, assessed using HADS. Changes in HADS total and subscale scores over time were estimated by using mixed factorial analysis of variance for repeated measurements. Additionally, a logistic regression model was used to investigate associations between HADS-D and HADS-A subscale scores of $\geq 8$ at baseline and prespecified covariates (such as age, sex, time since first diagnosis, type of DMT and level of disease-related distress) and other variables. An exploratory analysis examined score changes in patients with a baseline HADS score indicative of depression and anxiety versus patients with HADS-D or HADS-A subscale scores of $<8$ or HADS total scores of $<16$. Changes in HDRS, SF-36 version 2 and IES scores over time were also assessed, using mixed factorial analysis of variance for repeated measurements. To ensure consistency with other published trials in MS, items on the SF-36 were scored using the original 0-100 algorithms, instead of the norm-based scoring algorithms.

Primary and secondary objective analyses were conducted in the per-protocol population, comprising all included patients with all assessments (completers), independently from protocol violations. For all analyses, p-values of $<0.05$ were considered statistically significant.

Based on previous experience [8], it was expected that with a decrease in the HADS total score of 1 point at 12 months (with a standard deviation of 5 points), it would be possible to calculate an effect size of 0.20 . A sample size of 200 patients would have power of $80 \%$ with a significance level of 0.05 (two-sided test) to demonstrate an effect size of 0.20 . Allowing for a drop-out rate of $20 \%$, a total of 250 patients had to be enrolled.

\subsubsection{Missing data}

Unless otherwise stated, patients with missing data were not considered in the calculations of percentages. For analysis of the primary and main secondary endpoint, where the total or the subtotals of the rating scales was missing owing to missed answers to less than the $50 \%$ of the items, the totals or subtotals have been calculated by the mean of the filled items. Missing totals or subtotals of the rating scale owing to a non-monotonic pattern (i.e. missing value at visit 2 but present data at visit 1 and 3 ) were determined by interpolation between the values present at the two adjacent visits. For the HADS total and subscales, the analysis was performed via SAS PROC MIXED by fitting general linear and random coefficients general linear models with several variance-covariance matrix patterns (e.g. variance components, 
compound symmetry, unstructured, first order autoregressive). For the other scales (and subscales) the analyses was performed for all patients with complete data (at all three visits) by mean of SAS PROC GLM (general linear model).

\section{Results}

\subsection{Study participants}

A total of 250 patients were enrolled in the study and 222 (88.8\%) completed the study, representing the per-protocol population. Of the $28(11.2 \%)$ patients who did not complete the study, 4 were lost to follow-up, 2 discontinued because of adverse events, 1 withdrew consent and 'other' reasons were recorded for the remaining 21 patients. Patient demographic data and baseline disease characteristics are shown in Table 1 . Baseline characteristics were generally similar between women and men, although there was a trend for younger age of disease onset in women (mean age at diagnosis of 34.84 years in women and 37.38 years in men; $p=0.064$ ). Mean baseline score of the Hamilton Depression Rating Scale (HDRS) and of the total score and subscales of the HADS were below the cut-off score for anxiety and depression. The mean HADS-A score ( $6.20 \pm 3.99$ vs. $4.22 \pm 3.41$; $\mathrm{p}=0.007)$ and the mean total HADS score ( $12.27 \pm 6.10$ vs. $9.94 \pm$ $5.21)$ were statistically significantly higher in women than in men. At baseline only $27 \%$ of patients had a HADS-A score $\geq 8$ and $23 \%$ a HADS-D score $\geq 8$. Patients included in the study were at an initial disease stage with mild symptoms. Out of the 250 included patients, 219 had a disease duration shorter than one year, 11 patients between one and two years. Twenty 20 patients had a disease longer than 2 years but had not previously received DMT. Expanded Disability Status Scale (EDSS) scores at baseline are shown in Table 1. EDSS score remained stable over the course of the study.

All patients started treatment with a single DMT (Table 2). Approximately half of the patients received glatiramer acetate and approximately half received interferon- $\beta$ ( 47.2 and $51.2 \%$, respectively). The majority of patients received DMT without modification during the study. A total of 25 (10\%) patients had at least one DMT discontinuation between baseline and visit 2 (total of 29 discontinuations) and 25 (10\%) patients had at least one DMT discontinuation between visit 2 and visit 3 (total of 26 discontinuations). Among these patients, only 15 did not restart DMT during the study and of these, 10 withdrew from the study. In the remaining cases the same DMT was resumed or another DMT was started.

Table 1

Baseline demographic and disease characteristics.

\begin{tabular}{ll}
\hline Characteristic & \\
\hline Gender, $\mathrm{n}(\%)[\mathrm{n}=250]$ & $186(74.4)$ \\
$\quad$ Female & $64(25.6)$ \\
$\quad$ Male & $36.41(9.43)$ \\
Age, years [n=250] & $36.00(18.00-60.00)$ \\
$\quad$ Mean (SD) & \\
$\quad$ Median (range) & $33.31(9.07)$ \\
Age at first MS symptom, years [n = 245] & $31.74(16.68-58.71)$ \\
$\quad$ Mean (SD) & \\
Median (range) & $35.49(9.48)$ \\
Age at MS diagnosis [n $=249]$ & $34.87(17.81-58.98)$ \\
$\quad$ Mean (SD) & \\
Median (range) & $35.94(9.26)$ \\
Age at last MS exacerbation [n = 235] & $35.73(17.87-58.71)$ \\
Mean (SD) & \\
Median (range) & $1.58(1.06)$ \\
EDSS total score [n $=244]$ & $1.50(0.00-5.50)$ \\
$\quad$ Mean (SD) & 242 \\
Median (range) & 2 \\
Mild disability (EDSS 0-4.5), $\mathrm{n}$ & \\
Moderate disability (EDSS 5.0-6.5), $\mathrm{n}$ &
\end{tabular}

EDSS, Expanded Disability Status Scale; MS, multiple sclerosis.
Table 2

Type of disease-modifying treatment (DMT) prescribed initially and after discontinuation, values are $\mathrm{n}(\%)$.

\begin{tabular}{|c|c|c|}
\hline \multicolumn{2}{|l|}{ Type of treatment initially prescribed } & $(\mathrm{n}=250)$ \\
\hline \multicolumn{2}{|l|}{ Glatiramer acetate (Copaxone $®)$} & $118(47.2)$ \\
\hline \multicolumn{3}{|l|}{ Interferon- $\beta-1 \mathrm{a}$} \\
\hline \multicolumn{2}{|l|}{ Avonex ${ }^{\circledR}$} & $49(19.6)$ \\
\hline \multicolumn{2}{|l|}{ Rebif® 22} & $29(11.6)$ \\
\hline \multicolumn{2}{|l|}{ Rebif® 44} & $17(6.8)$ \\
\hline \multicolumn{2}{|l|}{ Rebif $\circledast 22+44$} & $6(2.4)$ \\
\hline \multicolumn{3}{|l|}{ Interferon- $\beta-1 \mathrm{~b}$} \\
\hline \multicolumn{2}{|l|}{ Betaferon ${ }^{\circledR}$} & $19(7.6)$ \\
\hline \multicolumn{2}{|l|}{ Extavia ${ }^{\circledR}$} & $8(3.2)$ \\
\hline \multicolumn{2}{|l|}{ Azathioprine } & $2(0.8)$ \\
\hline \multicolumn{2}{|l|}{ None $^{\mathrm{a}}$} & $2(0.8)$ \\
\hline \multicolumn{3}{|l|}{ Discontinuation of DMT treatment } \\
\hline Patients with one DMT discontinuation & 25 & 25 \\
\hline Total number of discontinuations & 29 & 26 \\
\hline Discontinuation of DMT ${ }^{\mathrm{b}}$ & Visit 2 & Visit 3 \\
\hline DMT restarted & $3(10.3)$ & $6(23.1)$ \\
\hline New DMT started & $21(72.4)$ & $10(38.5)$ \\
\hline No new DMT started & $5(17.2)$ & $10(38.5)$ \\
\hline New treatment & Visit 2 & Visit 3 \\
\hline Avonex® & $6(28.6)$ & $1(10.0)$ \\
\hline Azathioprine & $1(4.8)$ & $0(0)$ \\
\hline Betaferon $®$ & $2(9.5)$ & $0(0)$ \\
\hline Copaxone ${ }^{\circledR}$ & $6(28.6)$ & $3(30.0)$ \\
\hline Rebif® 22 & $1(4.8)$ & $2(20.0)$ \\
\hline Rebif® 44 & $5(23.8)$ & $3(30.0)$ \\
\hline Tysabri® & $0(0)$ & $1(10.0)$ \\
\hline
\end{tabular}

${ }^{a}$ These patients dropped out of the study, did not receive any treatment and were not included in the per-protocol analysis.

b Percentages are calculated on number of interruptions.

Adverse events, lack of efficacy and 'other' were the reason for discontinuation in 11, 9 and 9 patients, respectively, between baseline and visit 2, and in 11, 6 and 9 patients, respectively, between visit 2 and visit 3. During the study, at least one additional concomitant medication was administered to 134 patients. Corticosteroids for MS relapse were administered to almost $30 \%$ of patients, with methylprednisolone administered in $18.5 \%$ of cases.

\subsection{Primary objective}

At baseline, mean HADS total and HADS-A and HADS-D subscale scores were within the normal range (Table 3). There were no significant changes over time in mean HADS total and HADS-A and HADS-D subscale scores (Table 3 ). There was no statistically significant interaction between changes in HADS scores over time and the two main types of DMT (glatiramer acetate and interferon- $\beta$ ) [p-value for interaction $=0.1677$.

Logistic regression analysis demonstrated that, at baseline, the SF-36 vitality $(\mathrm{p}<0.0001)$ and mental health $(\mathrm{p}=0.0061)$ domains were independent predictors of a HADS-D subscale score of $\geq 8$. For the HADS-A subscale, logistic regression analysis found that independent predictors at baseline that were significantly associated with a HADS-A subscale score of $\geq 8$ were the SF-36 role-physical ( $p=0.0522$ ), vitality ( $p=$ $0.013)$ and mental health $(\mathrm{p}<0.0001)$ domains, age at diagnosis $(\mathrm{p}=0.0231)$ and the IES-R total score $(\mathrm{p}=0.0009)$.

Exploratory analysis revealed that patients with baseline scores indicative of anxiety or depression (HADS total score of $\geq 16$ [ $n=46]$, HADS-A subscale score of $\geq 8$ [ $n=55$ ] or HADS-D subscale score of $\geq 8$ $[\mathrm{n}=46]$ ) tended to improve over time (Table 4).

Patients with baseline scores in the normal range (HADS total score of $<16$ [ $n=155$ ], HADS-A subscale score of $<8$ [ $n=146$ ] or HADS-D subscale score of $<8$ [ $\mathrm{n}=155]$ ) tended to worsen over time (Table 4 ) although remaining within the normal range. The time-by-group interaction was statistically significant $(\mathrm{p}<0.0001)$ for the HADS total score and the HADS-D and HADS-A subscale scores. 
Table 3

Changes in patient-reported outcomes over time.

\begin{tabular}{|c|c|c|c|c|}
\hline & Baseline & Visit 2 & Visit 3 & Between all times p-value \\
\hline \multicolumn{5}{|l|}{ HADS scores $[\mathrm{n}=201]$} \\
\hline \multicolumn{5}{|l|}{ HADS total score } \\
\hline Mean (SD) & $11.60(6.10)$ & $11.38(6.20)$ & $11.06(6.02)$ & \multirow[t]{2}{*}{0.5050} \\
\hline Mean change from baseline (SD) & $11.60(6.10)$ & $0.22(4.77)$ & $0.53(5.66)$ & \\
\hline \multicolumn{5}{|l|}{ HADS anxiety subscale score } \\
\hline Mean (SD) & $5.58(3.96)$ & $5.46(4.12)$ & $5.23(3.90)$ & \multirow[t]{2}{*}{0.5063} \\
\hline Mean change from baseline (SD) & & $0.11(3.11)$ & $0.34(3.65)$ & \\
\hline \multicolumn{5}{|l|}{ HADS depression subscale score } \\
\hline Mean (SD) & $6.02(2.73)$ & $5.91(2.60)$ & $5.83(2.69)$ & \multirow[t]{2}{*}{0.6903} \\
\hline Mean change from baseline (SD) & & $0.11(2.30)$ & $0.19(2.62)$ & \\
\hline \multicolumn{5}{|l|}{ HDRS scores } \\
\hline \multicolumn{5}{|l|}{ HDRS total score $[\mathrm{n}=214]$} \\
\hline Mean (SD) & $6.77(5.96)$ & $5.88(5.81)$ & $5.63(5.77)$ & \multirow[t]{2}{*}{0.0032} \\
\hline Mean change from baseline (SD) & & $0.89(4.42)^{\mathrm{a}}$ & $1.14(5.67)^{\mathrm{a}}$ & \\
\hline \multicolumn{5}{|l|}{ IES-R total score $[\mathrm{n}=198]$} \\
\hline Mean (SD) & $1.15(0.76)$ & $1.03(0.79)$ & $0.96(0.78)$ & \multirow[t]{2}{*}{0.0009} \\
\hline Mean change from baseline (SD) & & $0.12(0.66)^{\mathrm{b}}$ & $0.18(0.73)^{\mathrm{b}}$ & \\
\hline
\end{tabular}

\subsection{Secondary objectives}

Both the HDRS and IES-R total scores improved over time, with statistically significant differences seen between all times, between baseline and visit 2 and between baseline and visit 3 (Table 3). No significant changes were seen over time for the SF-36 domains of physical functioning, role-physical, bodily pain, general health, vitality, social functioning and role-emotional. Changes over time in the SF-36 mental health domain did not show statistical significance $(\mathrm{p}=0.0682)$.

\subsection{Adverse events}

DMT was generally well tolerated. There was at least one adverse event reported in 126 (50.4\%) patients and at least one serious adverse event reported in 6 (2.4\%) patients. No deaths were reported during the study.

Discontinuation because of adverse events occurred in 24 (9.6\%) patients and discontinuation because of serious adverse events occurred in $2(0.8 \%)$ patients. The most commonly reported adverse events included MS-related symptoms (27.6\%), influenza (14.4\%), injection-site reactions (7.6\%) and asthenia (4.4\%). Adverse events considered related to

\section{Table 4}

Exploratory analyses of HADS scores over time.

\begin{tabular}{lccc}
\hline & Baseline & Visit 2 & Visit 3 \\
\hline $\begin{array}{l}\text { Patients with baseline scores indicative of anxiety or depression }{ }^{\mathrm{a}} \text { tended to } \\
\text { improve }\end{array}$ & $20.73(4.28)$ & $18.52(6.16)$ & $16.67(5.94)$ \\
$\begin{array}{l}\text { HADS total scores } \\
\text { HADS-A subscale scores } \\
\quad \text { Mean (SD) }\end{array}$ & $10.94(2.49)$ & $9.30(4.10)$ & $8.18(3.39)$ \\
$\begin{array}{l}\text { HADS-D subscale scores } \\
\text { Mean (SD) }\end{array}$ & $10.11(2.22)$ & $8.52(2.92)$ & $8.17(3.30)$ \\
$\begin{array}{l}\text { Patients with baseline scores in the normal range } \\
\text { HADS total scores }\end{array}$ & $8.88(3.27)$ & $9.26(4.36)$ & $9.40(4.95)$ \\
$\begin{array}{l}\text { HADS-A subscale scores } \\
\quad \text { Mean (SD) }\end{array}$ & $3.55(2.05)$ & $4.01(3.06)$ & $4.12(3.48)$ \\
$\begin{array}{l}\text { HADS-D subscale scores } \\
\text { Mean (SD) }\end{array}$ & $4.80(1.30)$ & $5.11(1.86)$ & $5.13(2.01)$ \\
\hline
\end{tabular}

HADS, Hospital Anxiety and Depression Scale; SD, standard deviation time by group interaction $(\mathrm{p}<0.0001)$.

a HADS total score of $\geq 16(n=46)$, HADS-A subscale score of $\geq 8(n=55)$ or HADS-D subscale score of $\geq 8(n=46)$.

b HADS total score of $<16(n=155)$, HADS-A subscale score of $<8(n=146)$ or HADSD subscale score of $<8(n=155)$.
DMT occurred in 66 patients (93 events in total). Out of these, only twenty-six events were considered to be related to glatiramer acetate; local injection-site reactions accounted for 17 of these 26 events, with only one serious adverse event (severe hepatitis) considered to be related to glatiramer acetate.

\section{Discussion}

This study was aimed to investigate, in an observational period of 12 months, the change of feeling of anxiety and depression measured by the HADS over the time, in order to evaluate the impact of DMT treatment on emotional burden of recently diagnosed MS patients.

Depression and anxiety are often present at the time of diagnosis in patients with MS. A previous study indicated that anxiety was prominent in the period surrounding the diagnosis of MS, and that HADS-A scores, but not HADS-D scores, significantly improved over time [8]. In this study $43 \%$ of patient had a baseline HADS-A score $\geq 8$ and only $11 \%$ a baseline HADS-D score $\geq 8$.

In the POSIDONIA study, baseline HADS total, HADS-A and HADS-D subscale scores were below the cut-off scores for anxiety and depression, $27 \%$ and $23 \%$ of patients having a baseline score $\geq 8$ for the HADS-A and HDS-D, respectively. Baseline scores for HDRS indicated that patients had very mild depressive symptoms, with baseline scores for IES-R and SF-36 also supporting the presence of only low level impairment. The fact that mean HADS scores were normal at baseline limited the likelihood of detecting an improvement in depression and anxiety following the introduction of DMT. Indeed, overall, HADS total and HADS-A and HADS-D subscale scores did not significantly change over time. Although largely used in the literature, the choice of the HADS as primary outcome measure may have not been optimal. The HADS is an instrument with good psychometric properties in terms of factor structure, sub-scale intercorrelation, homogeneity and internal consistency. However it was developed as a screening instrument and its simple scoring does not allow enough room for change detection, particularly in the presence of very mild symptoms (floor effect). Indeed, when comparing the change over time in patients with pathological levels of anxiety or depression as opposed to non-anxious and nondepressed patients the positive influence of starting DMT becomes more evident. This finding however needs to be confirmed in a larger sample.

Moreover, significant improvements were seen over time in the secondary objectives of HDRS total scores and IES-R total scores. The fact that the HDRS - a more complex and wider scale - was more sensitive to change seems to support the above consideration. 
In general, no significant changes over time were seen in SF-36 domain scores, although the change in the mental health domain score was of borderline statistical significance. Overall, DMT was generally well tolerated in the POSIDONIA study.

Data are limited concerning the effect of DMT on emotional burden in patients with recently-diagnosed MS. While there has been concern that immunomodulatory treatment may induce depression [11], rigorous studies have failed to demonstrate an association between interferon- $\beta$ or glatiramer acetate and depression [11]. Regarding the possibility of an improvement in depression and anxiety symptoms with DMT, data from the current study suggest such treatment may have a positive role to play. Although the POSIDONIA study did not meet the primary objective owing to methodological limitations and to the observational nature of the study, it provides a valuable background for future research.

\section{Conclusions}

In the patient population of the POSIDONIA study depression and anxiety were present in a minority of patients thus not allowing to detect the impact of starting DMT. However DMT appears to have a positive effect in patients with measurable anxiety or depression at baseline.

DMT was generally well tolerated in patients with recentlydiagnosed MS.

\section{Conflicts of interest}

E. Montanari received grants from Novartis and Serono, and speaker honoraria from Biogen and Teva. D. Maimone received speaker honoraria and travel grants from Bayer Healthcare, Biogen Italia, Merck Serono, Novartis, Genzyme, and Teva pharmaceuticals. P. Confalonieri was a Board member for Biogen Idec, received support for conference travel from Sanofi-Aventis, Biogen Dompé AG and Merk Serono. M. Frigo has been involved in Advisory Boards organized by Biogen Idec and Novartis and in clinical trials sponsored by Teva, Merck Serono and Almirall. A. Francia has been involved in activities sponsored by Teva, Novartis, Biogen Idec, Sanofi, Genzyme, Merck Serono, CSL Behring. P. Ragonese received travel expenses or honoraria for consultancies or advisory boards from Biogen Idec, Genzyme Sanophi, Merck Serono, Novartis, and TEVA pharmaceuticals. A.Veneziano is an employee of Teva Italia S.r.l. M. Rottoli, K. Plewnia, A. Pala, N. A. Losignore declare no conflicts of interest.

\section{Acknowledgments}

We would like to thank Gillian Keating of Springer Healthcare Communications and Marie Cheeseman who provided medical writing assistance on behalf of Springer Healthcare Communications. This medical writing assistance was funded by TEVA Italia, Italy. We would like to thank Maria Cristina Jori of Mediolanum Cardio Research for support in data analysis.
Finally, the authors would also like to thank all other members of the POSIDONIA study group: Dr. Sebastiano Traccis, Dr. Giovanni Bosco Zimatore, Dr. Michele Dotta, Prof. Sauro Severi, Dr. Michele Ragno, Dr. Rosa Maria Candeago, Dr. Michele Feleppa, Dr. Sergio Stecchi, Dr. Domenico Bosco, Prof. Maria Rosaria Tola, Prof. Luca Massacesi, Prof. Giancarlo Costantino, Dr. Claudio Marcello Solaro, Prof. Carlo Serrati, Dr. Rocco Totaro, Dr. Maria Gabriella Coniglio, Prof. Elio Scarpini, Dr. Cristina Zuliani, Dr. Franco Perla, Dr. Anna Ticca, Dr. Salvatore Cottone, Prof. Alfonso Iudice, Prof. Tatiana Koudriavteva, Prof. Giancarlo Di Battista, Prof. Claudio Gasperini, Dr. Chiara Perin, Prof. Giovanni Meola, Prof. Maura Pugliatti, Prof. Fabio Bandini, Dr. Paola Cavalla, Dr. Federico Posteraro, and Dr. Domenico Consoli for their involvement in the study.

\section{References}

[1] Multiple sclerosis: management of multiple sclerosis in primary and secondary care. National Institute for Health and Care Excellence, 2014. Available at: http://www. guidance.nice.org.uk/cg186 [Last accessed.

[2] R.A. Marrie, S. Reingold, J. Cohen, et al., The incidence and prevalence of psychiatric disorders in multiple sclerosis: a systematic review, Mult. Scler. 21 (2015) 305-317.

[3] A.C. Janssens, D. Buljevac, P.A. van Doorn, et al., Prediction of anxiety and distress following diagnosis of multiple sclerosis: a two-year longitudinal study, Mult. Scler. 12 (2006) 794-801.

[4] R.F. Brown, E.M. Valpiani, C.C. Tennant, et al., Longitudinal assessment of anxiety, depression, and fatigue in people with multiple sclerosis, Psychology and Psychotherapy 82 (2009) 41-56.

[5] O.P. Dahl, E. Stordal, S. Lydersen, R. Midgard, Anxiety and depression in multiple sclerosis. A comparative population-based study in Nord-Trondelag County, Norway, Mult. Scler. 15 (2009) 1495-1501.

[6] A. Feinstein, S. Magalhaes, J.F. Richard, et al., The link between multiple sclerosis and depression, Nat. Rev. Neurol. 10 (2014) 507-517.

[7] A. Giordano, G. Ferrari, D. Radice, et al., Self-assessed health status changes in a community cohort of people with multiple sclerosis: 11 years of follow-up, Eur. J. Neurol. 20 (2013) 681-688.

[8] A. Giordano, F. Granella, A. Lugaresi, et al., Anxiety and depression in multiple sclerosis patients around diagnosis, J. Neurol. Sci. 307 (2011) 86-91.

[9] K.H. Jones, D.V. Ford, P.A. Jones, et al., A large-scale study of anxiety and depression in people with multiple sclerosis: a survey via the web portal of the UK MS Register, PLoS One 7 (2012), e41910.

[10] S.L. Minden, A. Feinstein, R.C. Kalb, et al., Evidence-based guideline: assessment and management of psychiatric disorders in individuals with MS: report of the Guideline Development Subcommittee of the American Academy of Neurology, Neurology 82 (2014) 174-181.

[11] J.J. Vattakatuchery, H. Rickards, A.E. Cavanna, Pathogenic mechanisms of depression in multiple sclerosis, J. Neuropsychiatry Clin. Neurosci. 23 (2011) 261-276.

[12] K.H. Jones, P.A. Jones, R.M. Middleton, et al., Physical disability, anxiety and depression in people with MS: an internet-based survey via the UK MS Register, PLoS One 9 (2014), e104604.

[13] A.C. Janssens, P.A. van Doorn, J.B. de Boer, et al., Impact of recently diagnosed multiple sclerosis on quality of life, anxiety, depression and distress of patients and partners, Acta Neurol. Scand. 108 (2003) 389-395.

[14] L. Del Piccolo, E. Pietrolongo, D. Radice, et al., Patient expression of emotions and neurologist responses in first multiple sclerosis consultations, PLoS One 10 (2015), e0127734.

[15] C.H. Polman, S.C. Reingold, B. Banwell, et al., Diagnostic criteria for multiple sclerosis: 2010 revisions to the McDonald criteria, Ann. Neurol. 69 (2011) 292-302.

[16] K. Honarmand, A. Feinstein, Validation of the hospital anxiety and depression scale for use with multiple sclerosis patients, Mult. Scler. 15 (2009) 1518-1524. 